\title{
Grape Seed Polyphenolic Extract as a Potential Therapeutic Compound in Tau- Mediated Neurodegenerative Disorders. Electron Microscopy Analysis of Paired Helical Filaments.
}

\author{
I. Santa-Maria* \\ * Department of Neurology, Mount Sinai School of Medicine. New York, NY10029.
}

Compelling evidence strongly supports the hypothesis that accumulation of misfolded proteins leads to synaptic dysfunction, brain damage, and disease [1]. Abnormal misfolding of the microtubuleassociated protein, tau, leads to aggregation of tau into paired helical filaments that are ultimately deposited as intracellular neurofibrillary tangles in the brain. Neurofibrillary tangle neuropathology is a key feature among a number of neurodegenerative disorders, including Alzheimer's disease, Progressive supranuclear palsy, Corticobasal degeneration, Argyrophilic grain disease, Pick's disease, as well as a number of familial Frontotemporal dementia with Parkinsonism linked to chromosome 17 , that are collectively referred to as tauopathies. We have been previously described that a selected grape seed polyphenolic extract (GSPE) may attenuate the generation and stability of Alzheimer's disease like $\beta$-amyloid peptide aggregates $[2,3]$. That indicates that certain grapederived dietary compounds - in particular, certain polyphenolic compounds enriched in grape derived polyphenols - may interfere with abnormal protein folding, thereby reducing the accumulation of neurotoxic proteins. Based on our recent observation that a particular GSPE attenuates the generation and stability of misfolded proteins $[2,3]$ we hypothesized that this GSPE might also attenuate the tau protein misfolding that leads to the generation of tau aggregates critical for the initiation and progression of neurodegeneration and/or cognitive dysfunctions in tauopathies. In this way, we have found that GSPE significantly inhibits self-aggregation of a synthetic tau peptide and promotes dissociation of preformed tau peptide aggregates, suggesting that GSPE might be able to beneficially modulate tau-mediated neuropathology [4]. The goal of our present studies was to examine a direct effect of GSPE on dissociation and ultrastructural characteristics of paired helical filaments isolated from patients with various tauopathies (Fig. 1). We found that GSPE treatment profoundly alters ultrastructural characteristics of paired helical filaments (Fig. 2) and reduces their quantity. Moreover, GSPE causes a significant disintegration of paired helical filaments in the presence of trypsin. Our results indicate that GSPE could be responsible for dissociation/disintegration of abnormal tau filaments with or without trypsin. Based on our findings, we hypothesized that GSPE might promote dissociation of misfolded tau fibrillary aggregates in the human brains. Evidence from this study supports the development of GSPE for the prevention and/or treatment of tau-mediated neurological disorders.

\section{References}

[1] C. Soto \& L.D. Estrada, Arch. Neurol. 65 (2) (2008) 184.

[2] J. Wang et al., J. Neurosci. 28 (2008) 6388.

[3] K. Ono et al., J. Biol. Chem. 283 (2008) 32176.

[4] L. Ho et al., J. Alzh. Dis. 16 (2009) 433. 


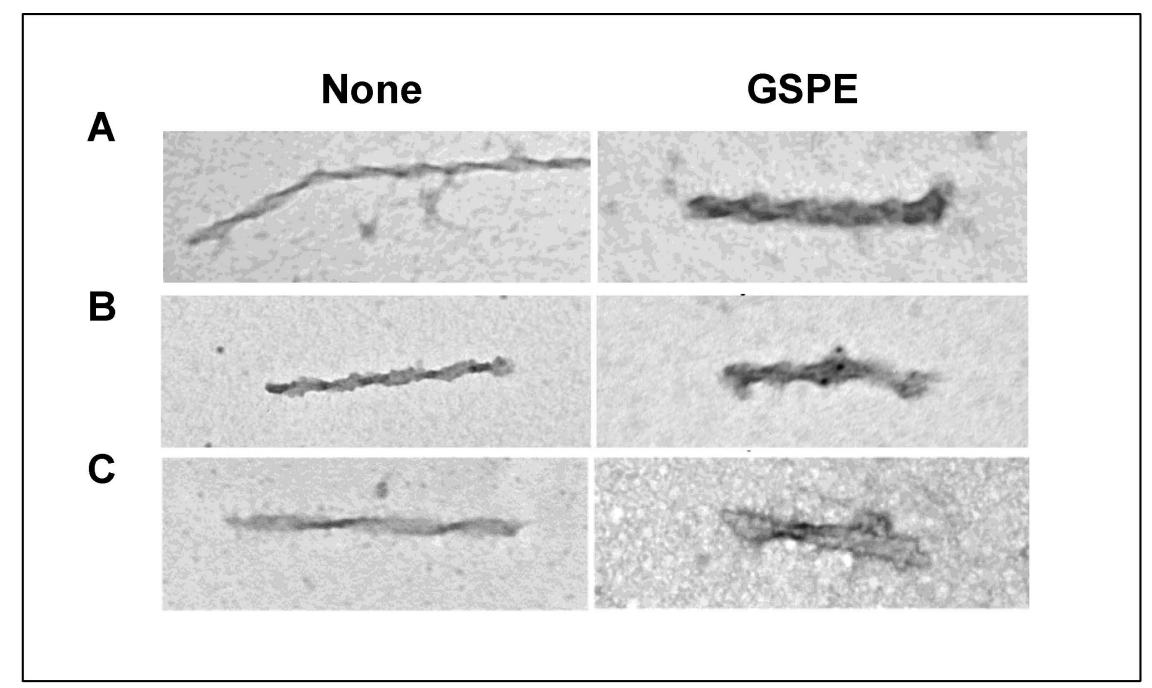

FIG. 1. GSPE promote ultrastructural changes in paired helical filaments from different tauopathies. Paired helical filaments (PHFs) were isolated from brain of patients with Alzheimer's disease (A), Progressive supranuclear palsy (B) and Corticobasal degeneration (C). GSPE treatment during 1 hour provokes ultraestructural alterations and promotes disintegration of PHFs.

No GSPE

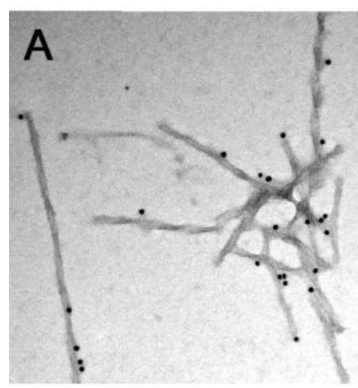

GSPE 5 sec

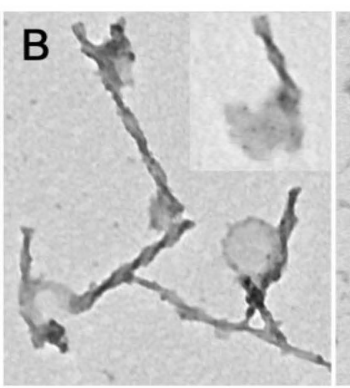

GSPE 1h

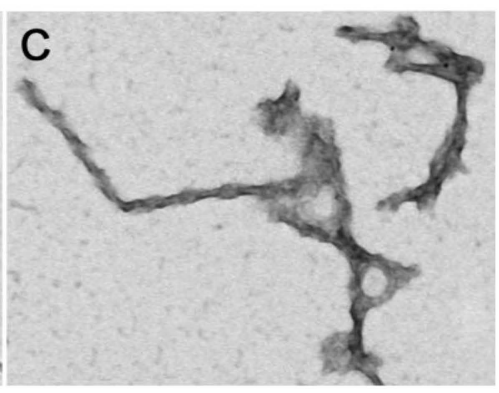

GSPE 24h

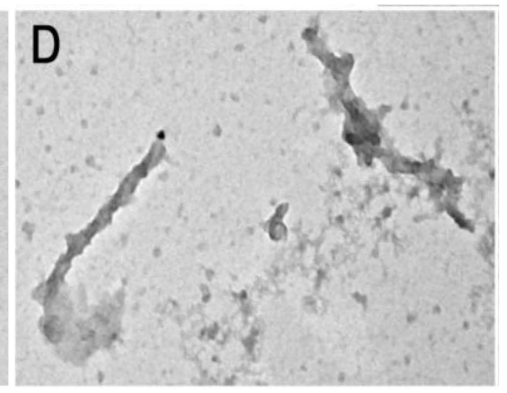

FIG. 2. GSPE reduces the stability and promotes morphological changes in PHFs from Alzheimer's disease. PHFs were isolated from patients with AD, subjected to treatment with GSPE $100 \mu \mathrm{M}$ during different times; $5 \mathrm{sec}(\mathrm{B}), 1$ hour (C), and 24 hours (D). 24 hours after treatment with GSPE almost a majority of PHFs have been disintregated due to a big disruption in the structure and stability of these filaments. 